INNOVATIONS IN PRIMARY CARE

\title{
A Staff- and Protocol-Driven System to Apply Urine Drug Testing in Primary Care
}

\author{
Ryan Patchett-Marble, $M D^{1,2}$ \\ 'Department of Family Medicine, Northern Ontario School of \\ Medicine, Thunder Bay, Ontario, Canada \\ ${ }^{2}$ Marathon Family Health Team, Marathon, Ontario, Canada \\ Ann Fam Med 2020;18:563. https://doi.org/10.1370/afm.2604.
}

\section{THE INNOVATION}

The HARMS Program (High-Yield Approach to Risk Mitigation and Safety) is the first of its kind-a complete primary care system built to support routine urine drug testing in chronic pain patients prescribed opioids. The program addresses previous barriers to testing through several innovative components: an intuitive and versatile clinical algorithm that guides frequency of testing and how to manage the full spectrum of results; automated testing interpretation and recommendations to prevent clinician errors; and a program structure that greatly reduces physician resource requirements through formal delegation of nearly all aspects of the program to non-medical staff (Supplemental Figures 1-3).

\section{WHO \& WHERE}

The Marathon Family Health Team is a rural primary care practice located in Marathon, Ontario, Canada (population 3,273). We are composed of family physicians, nurses, a physician assistant, and social worker. Our region of Northwestern Ontario has the highest rates in the province of opioid prescribing for pain, opioid addiction, and opioid-related deaths. ${ }^{1}$

\section{HOW}

We were aware of guideline recommendations supporting routine urine drug testing in chronic-pain patients, but like most North American physicians, we were not using it. Our barriers were similar to those reported in the literature: confusion around which type(s) of testing to use and at what frequency; challenges around correct testing interpretation and how to act on the results; and physician time and human resource concerns.

Conflicts of interest: author reports none.

\section{Corresponding author}

Ryan Patchett-Marble, MD

Marathon Family Health Team

22 Peninsula Rd

PO Box 399

Marathon, ON, Canada, POT 2EO

rmarble@nosm.ca
The first innovation we developed to address these barriers was a simple clinical approach that would guide not only how often to conduct testing for a given patient, but also how to take action for the full spectrum of results (Supplemental Figure 1). Initial risk assessment guides where the patient starts on the ladder, and new information (including a testing result) guides how a patient moves on the ladder. By tailoring the monitoring frequency and prescribing interval to a patient's level of risk-and dynamically adjusting that risk based on monitoring results-the risk ladder utilizes risk mitigation principles originally developed for safer opioid prescribing in opioid maintenance therapy.

The second innovation was developed to avert the potentially serious consequences that can arise from misinterpretation of test results. Called START-IT (Self-Report, Testing, and Automated Reading Tool for Immunoassay Tests), the program uses a tablet personal computer to collect all of the required information for an immunoassay test, and then uses best evidence to automatically interpret the results and make recommendations, including when to send for confirmatory testing (Supplemental Figure 2).

Our third innovation was to structure the program such that non-medical staff conduct nearly the entire program-from patient master list creation and maintenance, to testing randomization, booking, collection, immunoassay analysis, and automated interpretation (Supplemental Figure 3).

Early evidence suggests the program is effective at identifying patients being harmed by their opioid prescription and altering management (including starting treatment for opioid addiction). Given that the program was built and implemented in a resource-limited setting, it also offers a highly scalable option to other primary care sites interested in incorporating routine urine drug testing into their clinical practices.

\section{LEARNING}

A system to support routine urine drug testing in chronic pain patients prescribed opioids can be implemented in a primary care setting with minimal resources. The innovative clinical tools we developed as part of this system are freely available to other clinics interested in adapting them to their own setting (http://www.HARMSprogram.ca).

For additional information, including supplemental figures, funding support, prior presentations, and references, see https:/l www.annfammed.org/content/18/6/563/suppl/DC1/.

Key words: urine drug testing; opioid management; patient safety; Primary care; knowledge translation; systems development

Submitted April 26, 2019; submitted, revised, March 16, 2020; accepted March 24, 2020. 